\title{
LA ECONOMÍA SOCIAL EN CLAVE INTERNACIONAL. CUANTIFICACIÓN, RECONOCIMIENTO INSTITUCIONAL Y VISIBILIDAD SOCIAL EN EUROPA, IBEROAMÉRICA Y NORTE DE ÁFRICA
}

\author{
POR \\ Rafael CHAVES ÁVILA ${ }^{1}$, \\ Jose Luis MONZÓN CAMPOS ${ }^{2}$, \\ Jose María PÉREZ DE URALDE ${ }^{3}$ y \\ Mario RADRIGÁN ${ }^{4}$
}

\section{RESUMEN}

El presente trabajo tiene tres mayores objetivos: en primer lugar, pretende analizar el grado de visibilidad social del concepto de economía social desde una perspectiva internacional, entendiendo por ello su grado de conocimiento y percepción por la sociedad. En segundo lugar, estudiar su grado de institucionalización en el ordenamiento jurídico ya sea con leyes, políticas de fomento o con órganos específicos de atención a la economía social. Finalmente, pretende cartografiar cuantitativamente la economía social, trazando las dimensiones macroeconómicas de este tercer sector de las economías. El ámbito geográfico abordado son los países de la Unión Europea, Iberoamérica y Norte de África. Se parte de la

\footnotetext{
1 Rafael CHAVES ÁVILA. IUDESCOOP, Universitat de València. Dirección de correo electrónico: Rafael.Chaves@uv.es.

${ }^{2}$ Jose Luis MONZÓN CAMPOS. IUDESCOOP, Universitat de València. Dirección de correo electrónico: J.Luis.Monzon@uv.es.

${ }^{3}$ Jose María PÉREZ DE URALDE. GEZKI. Director del Observatorio Vasco de la Economía Social (OVESGEZKI) Universidad del País Vasco. Dirección de correo electrónico: director.observatorio.gezki@ehu.es

4 Mario RADRIGÁN. Universidad de Santiago de Chile. Dirección de correo electrónico: mario.radrigan@usach.cl.

Con la colaboración en el trabajo de campo de los investigadores y corresponsales nacionales que han participado en sendos estudios internacionales, en particular a Danièle Demoustier, Roger Spear, Chiara Carini, Magdalena Huncova, Alberto Zevi, para Europa, y Marietta Buchelli, Leandro Pereira, Ana Maria Dávila, Natalia García y Francesco Penaglia para Iberoamérica y Norte de África.
}

REVESCO No 112 - Extraordinario en Homenaje al Profesor Alfonso Carlos MORALES

GUTIÉRREZ - ISSN: 1885-8031 - www.ucm.es/info/revesco

http://dx.doi.org/10.5209/rev_REVE.2013.v112.43069

Fecha de recepción: 07/09/2013

Fecha de aceptación: 24/09/2013 
concepción de economía social acuñada en la literatura científica y recientemente sancionada en la nueva legislación en la materia.

Palabras clave: economía social, concepto, dimensiones económicas, Europa, Iberoamérica, África

Claves ECONLIT: D640, P130, Q130, L310

\title{
THE SOCIAL ECONOMY IN INTERNATIONAL PERSPECTIVE. ECONOMIC DIMENSIONS, INSTITUTIONAL RECOGNITION AND SOCIAL VISIBILITY IN EUROPE, LATINAMERICA AND NORTH OF AFRICA
}

\begin{abstract}
:
The present work has three major goals: first, it aims at analysing the degree of social visibility of the concept of social economy from an international perspective, understanding by social visibility their level of awareness and perception by the society.
\end{abstract}

Secondly, to study its degree of institutionalization in the legal framework either with laws, policies or building with specific bodies dedied to the social economy.

Keywords: social economy, concept, economic dimensions, Europe, Latinamerica, Africa

\section{INTRODUCCIÓN}

La economía social constituye una importante realidad social y económica, no siempre conocida ni reconocida, cuya actividad cotidiana silenciosa está mejorando las condiciones de vida y solucionando los problemas sustantivos de millones de personas en el mundo así como creando riqueza solidariamente.

Desde hace años la economía social está siendo objeto de atención por parte de la comunidad científica internacional, de la sociedad civil e incluso está emergiendo en la agenda política de numerosos gobiernos nacionales así como de instituciones internacionales tales como Naciones Unidas, la Organización de Estados Americanos, la Comisión Europea, la Organización para la Cooperación y el Desarrollo Económico y la Organización Internacional del Trabajo (Chaves y Demoustier, 2013). 
La plena incorporación de esta realidad social al imaginario social del mundo político, académico y de la sociedad civil organizada, como precondición para que adquiera el rol sociopolítico que le corresponde, pasa, a nuestro juicio, por dar respuesta a tres grandes retos:

1. el reto de la conceptualización y representación intelectual de esta realidad social, identificando sus características identitarias y consensuando una definición en el ámbito científico y en la sociedad;

2. el reto de su visibilidad social y de su reconocimiento institucional, esto es, por un lado, su grado de conocimiento y percepción por la sociedad, y por otro, su grado de institucionalización en el ordenamiento jurídico ya sea con leyes, políticas de fomento o con órganos específicos de atención a la economía social;

3. el reto de cartografiar cuantitativa y cualitativamente la economía social, tanto al objeto de valorar sus dimensiones macroeconómicas, de identificar experiencias paradigmáticas eventualmente reproducibles, como de medir su impacto social y económico;

El objeto del presente trabajo es analizar en qué medida la economía social se encuentra respondiendo a estos tres retos. Para este análisis nos beneficiamos de dos estudios de carácter internacional que hemos desarrollado en los últimos dos años y que han abordado sendas realidades, por un lado, la Unión Europea y por otro Iberoamérica y Norte de África. El primero ha sido dirigido por los profesores José Luis Monzón y Rafael Chaves por cuenta del Comité Económico y Social Europeo, en el que han participado en el trabajo de campo más de una treintena de corresponsales nacionales. El segundo estudio, elaborado para la Fundación Carolina, ha sido dirigido por los profesores Rafael Chaves y Jose María Pérez de Uralde y específicamente el trabajo empírico ha sido coordinado por el profesor Mario Radrigán. En este segundo estudio han participado también varios investigadores de sendas universidades iberoamericanas. En ambos casos han sido investigaciones empíricas pioneras que se han enfrentado a importantes problemas, sobre todo de disponibilidad y fiabilidad de información. 


\section{EL RETO DE LA CONCEPTUALIZACIÓN DE LA ECONOMÍA SOCIAL}

Precisar adecuadamente los contornos de la realidad "situada entre la economía pública y la economía capitalista", parafraseando a la obra seminal de Defourny y Monzón (1992) ha constituido el principal desafío en las pasadas dos décadas. Es sólo recientemente cuando este desafío ha encontrado una respuesta institucionalizada fuera del ámbito académico. En efecto, los artículos 3 y 4 de la propuesta de Ley española de fomento de la economía social realizada por el grupo de expertos dirigidos por el prof. José Luis Monzón cristalizaban una larga trayectoria de investigación científica en torno al concepto de economía social (Monzón, 2009). Ambos artículos establecen las señas identitarias de las entidades de economía social así como las formas jurídicas y empresariales concretas que se entendía respondían a tales señas de identidad. En concreto son las siguientes:

“Art. 3.- Principios de las entidades de Economía Social. Se consideran entidades de

la economía social todas aquellas de naturaleza privada cuyas reglas de funcionamiento

respondan a los siguientes principios:

a) Primacía de la persona y del objeto social sobre el capital

b) Adhesión voluntaria y abierta

c) Control democrático por parte de los miembros o socios

d) Conjunción de los intereses de los miembros y del interés general

e) Defensa y aplicación de los principios de solidaridad y responsabilidad

f) Autonomía de gestión e independencia respecto de los poderes públicos

g) Destino de la mayoría de los excedentes a la consecución de objetivos a favor del desarrollo sostenible, la mejora de los servicios a los miembros y el interés general

Art. 4.- Organizaciones y entidades de la Economía Social. A los efectos de la presente

Ley, se consideran entidades de la Economía Social, entre otras, las sociedades cooperativas, las mutuas y mutualidades, las sociedades laborales, las fundaciones privadas, las empresas sociales y, con carácter general, todas las asociaciones y entidades cuya organización y funcionamiento respondan principalmente a los principios formulados en el artículo anterior." (Monzón, 2009:147).

REVESCO No 112 - Extraordinario en Homenaje al Profesor Alfonso Carlos MORALES

GUTIÉRREZ - ISSN: 1885-8031 - www.ucm.es/info/revesco 
Con ligeras variaciones es esta concepción la recogida, en primer lugar, en la incipiente legislación europea en la materia, como la española Ley 5/2011, de 29 de marzo, de Economía Social, y la portuguesa Ley 30/2013, de 8 de mayo, Lei de Bases da Economía Social, en segundo lugar, por instituciones europeas como el Comité Económico y Social Europeo (en su Dictamen 01/10/2009 sobre "Diversidad de Formas de empresa" y en el Informe sobre la Economía Social en la Unión Europea realizado por CIRIEC por cuenta del Comité en 2012) y el Parlamento Europeo (Informe 'Toya' sobre Economía Social, 26/01/2009), y en tercer lugar por la entidad representativa de este sector socioeconómico a escala europea, Social Economy Europe, en su Carta de Principios de la Economía Social (2002), heredera, a su vez, de la tradición francesa de entidades representativas de la economía social que arranca a finales de los años setenta con el CNLMCA - Comité National de Liaison des Mutuelles, Coopératives et Associations-.

Desde la perspectiva científica, esta definición ha tenido que operativizarse de manera más precisa al objeto de ser útil en el ámbito de la obtención de macromagnitudes de contabilidad nacional y ello porque los dos sistemas de contabilidad nacional más importantes actualmente en vigor, el SCN-1993 de Naciones Unidas y el SEC-1995 de la Unión Europea, no contemplaban como un sector institucional diferenciado al conjunto de operadores económicos que integran la Economía Social. Ha sido sólo recientemente cuando la Comisión Europea ha realizado un Manual de orientación para la elaboración de una cuenta satélite de las empresas de la Economía Social (cooperativas y mutuas) (Monzón y Barea, 2007). En el Manual se ha integrado en un único concepto los principios históricos y valores propios de la Economía Social y la metodología de los citados sistemas de contabilidad nacional en vigor. A partir de los criterios establecidos por el Manual de la Comisión, el Informe ChavesMonzón del Comité Económico y Social Europeo ha formulado una definición operativa de Economía Social basada en un amplio consenso político y científico que está permitiendo cuantificar y hacer visible de forma homogénea y armonizada internacionalmente los principales datos agregados de las entidades que la integran. Dicha definición es la siguiente:

“Conjunto de empresas privadas organizadas formalmente, con autonomía de decisión y libertad de adhesión, creadas para satisfacer las necesidades de sus socios a través del mercado, produciendo bienes y servicios, asegurando o financiando y en las que la eventual distribución entre los socios de beneficios o excedentes así como la toma de decisiones, no están ligados directamente con el capital o cotizaciones aportados por cada socio, correspondiendo un voto a cada uno de ellos. La Economía Social también agrupa a aquellas entidades privadas organizadas

REVESCO No 112 - Extraordinario en Homenaje al Profesor Alfonso Carlos MORALES GUTIÉRREZ - ISSN: 1885-8031 - www.ucm.es/info/revesco 
formalmente con autonomía de decisión y libertad de adhesión que producen servicios de no mercado a favor de las familias, cuyos excedentes, si los hubiera, no pueden ser apropiados por los agentes económicos que las crean, controlan o financian” (Chaves, Monzón, 2008)

Esta definición, congruente con la formulada por los propios actores de la Economía Social, configura dos grandes subsectores de la misma, el de productores de mercado y el de productores no de mercado, clasificación que es muy útil para la elaboración de estadísticas fiables y la realización de análisis de la actividad económica, de conformidad con los sistemas de contabilidad nacional.

Dos ideas claves deben de ser puestas de relieve en relación al concepto de economía social. La primera radica en la prioridad puesta al elemento democrático y participativo de decisión como característica definitoria de las entidades de economía social y que le diferencia de otras concepciones intelectuales del llamado 'tercer sector'. La segunda idea a retener es que el concepto de economía social no es una definición por formas jurídicas (que incluirían tres o cuatro formas jurídicas básicas, a saber, las cooperativas, mutualidades, asociaciones con actividad económica y fundaciones) sino que es una definición de carácter estructural, es decir, una definición que establece características objetivas cuyo cumplimiento por parte de las diversas formas jurídicas les haría acreedoras de tal condición de entidad de economía social. Como conclusión de esta segunda idea, cada sociedad habrá construido sus propias formas jurídicas para dar cobertura a sus iniciativas sociales y económicas, con nexos en común a nivel internacional, y por tanto es necesaria la adaptación de aquel concepto a cada realidad nacional.

Ahora bien, no cabe duda que el concepto de economía social no ha sido la única construcción intelectual que abordara a 'aquel sector socioeconómico situado entre las administraciones- empresas públicas y las empresas privadas tradicionales de carácter capitalista" ni tampoco ha presentado un único sentido científico (Chaves, 1999). Al contrario, si bien puede considerarse que ha alcanzado un elevado grado de consenso internacional y social, no es menos cierto que continúa coexistiendo con otras tradiciones científicas, que llegan a ser preponderantes en algunos países, como la de las entidades no 
lucrativas (nonprofit organizations), la economía solidaria y la más reciente de las empresas sociales, entre muchas otras ${ }^{5}$.

\section{Otras concepciones}

- El enfoque del sector no lucrativo.

Este enfoque es de tradición eminentemente anglosajona siendo predominante en los Estados Unidos donde ha generado una abundante literatura científica. Ligado al mismo se encuentran también las locuciones de sector filantrópico, sector voluntario y sector benéfico, evidenciando la importancia de las charities británicas y las philanthropic foundations estadounidenses. La idea principal que caracteriza a las entidades no lucrativas es la forma de distribución de los excedentes o beneficios, basada en el Principio de no distribución de beneficios a las personas que las han creado o que las controlan o financian (Powell, 1987; Weisbrod, 1988).

En los años noventa, liderado por la Universidad Johns Hopkins de Estados Unidos, se precisó el concepto de organización sin fines de lucro, dentro de la tradición 'nonprofit', el cual permitió realizar un estudio comparativo internacional con la finalidad básica de ofrecer una panorámica, sobre todo cuantitativa, de este sector social.

Los cinco criterios de las organizaciones sin fines de lucro que se establecieron fueron (Salamon y Anheier, 1997):

a) son organizaciones, es decir, poseen una estructura y presencia institucional, generalmente con personalidad jurídica;

b) privadas, es decir, están institucionalmente separadas de los poderes públicos, aunque pueden recibir financiación pública e incluir en sus órganos directivos a funcionarios públicos;

\footnotetext{
${ }^{5}$ Es posible identificar más de una veintena de locuciones para referirse a este tercer sector en su sentido más amplio. Sin ser exhaustivos podemos citar: Economía social, Economía de entidades de participación, Sector no lucrativo, Empresas sociales, Tercer Sector, Sector voluntario, Sector filantrópico, Sector caritativo, Sector Cooperativo, Sector autogestionario, Economía solidaria, Economía Popular y Solidaria, Sector asociativo, Sector social, ONG's, Empresas socialmente responsables, 'Empresas B', entre otras. Coincidimos con Perri 6 y Pestoff (1993) en que las distintas locuciones y definiciones existentes constituyen construcciones políticas e intelectuales de proyectos políticos, en absoluto neutrales, no siempre comprendidos ni conscientemente utilizados en sus sesgos por el mundo académico y por la sociedad.
} 
c) autónomas, es decir, con capacidad de control sobre sus propias actividades y libertad para elegir y cesar a sus órganos de gobierno;

d) no reparten beneficios, es decir, estas entidades pueden generar beneficios pero éstos deben ser reinvertidos en la misión principal de la organización y no ser distribuidos entre sus propietarios, miembros fundadores u órganos directivos;

e) voluntarias, lo que significa, en primer lugar, que la pertenencia a ellas no es obligatoria ni está legalmente impuesta y, en segundo lugar, que deben contar con la participación de voluntarios en sus actividades u órganos de gestión.

\section{- El enfoque de la economía solidaria.}

Siguiendo a Monzón y Chaves (2012), con esta misma denominación han aparecido dos concepciones de economía solidaria, una europea y otra iberoamericana, con importantes nexos de unión. Ambas presentan como característica el objetivo de producir bienes sociales o preferentes para sus miembros o para la sociedad. Los bienes preferentes son aquellos sobre los cuales existe un amplio consenso político y social en cuanto a que son esenciales para una vida digna, y por lo tanto deben estar a disposición de toda la población, independientemente de su renta o poder adquisitivo. Cuando el gobierno se muestra incapaz de proveer a toda la población de estos bienes y servicios, es la propia sociedad civil la que se auto organiza para satisfacer sus necesidades. Es así como, en un contexto de reestructuración o insuficiencia de provisión de los Estados sociales, han emergido nuevas organizaciones sociales, junto a las clásicas cooperativas. Según el enfoque francés, esta 'nueva economía social y solidaria' presenta tres características distintivas: a) las demandas sociales a las que pretende dar respuesta, b) los actores que promueven estas iniciativas y c) el deseo explícito de un cambio social. Desde esta concepción la forma jurídica es poco relevante y el posicionamiento económico es híbrido, pues entiende que la economía solidaria si sitúa a caballo entre tres polos: el mercado, el estado y la reciprocidad, polos que se corresponden a los principios de mercado, redistribución y reciprocidad (Laville, 1994). En la práctica, las cooperativas y asociaciones son las formas dominantes de esta economía solidaria, aunque rechazan que lo sean todas, excluyendo, por ejemplo, a las cooperativas más institucionalizadas y 'banalizadas por el mercado' precisamente por su escaso carácter transformador social. 
En Iberoamérica la economía solidaria está más ligada al concepto de economía popular y hace mayor hincapié a la idea de constituir una fuerza de transformación social portadora de un proyecto de sociedad alternativa a la globalización neoliberal, respondiendo además en mayor medida a los problemas de pobreza, subdesarrollo y exclusión social (Singer, 1997, Coraggio, 1997).

\section{- El enfoque de las empresas sociales.}

Siguiendo a Monzón y Chaves (2012), una prolífica literatura científica ha aparecido en los últimos años versada sobre las empresas sociales, si bien carente de consenso en cuanto al contenido de este enfoque. Es posible, sin embargo, distinguir cuanto menos dos enfoques al respecto, el angloamericano y el europeo continental (Defourny y Nyssens, 2011).

El enfoque angloamericano es a su vez muy variado, incluyendo desde corrientes que consideran que las empresas sociales son las homólogas en el mercado de las organizaciones privadas sin fines de lucro con un propósito social, hasta a aquellas entidades orientadas exclusivamente a la innovación social y a la satisfacción de las necesidades sociales, siempre independientemente de su forma jurídica.

El enfoque europeo continental es heredero de los trabajos de la red EMES, que considera que las empresas sociales son el resultado del emprendimiento colectivo en la economía social y están definidas por tres bloques de indicadores (relacionados con la estructura económica, social y de gobierno). La Comisión Europea también define a las empresas sociales como un subgrupo de la economía social.

\section{EL RETO DE LA VISIBILIDAD SOCIAL DE LA ECONOMÍA SOCIAL}

Tras exponer las líneas maestras de la conceptualización de la economía social y de sus conceptos afines, interesa abordar a continuación el grado de conocimiento y de percepción social de estos términos y conceptos, conscientes de la existencia de confusiones en su utilización, de su desconocimiento y de la convivencia de varios conceptos a la vez en algunos países. Interesa, en primer lugar, evaluar el nivel de reconocimiento de la economía social en tres ámbitos relevantes: la administración pública, el mundo académico y científico y el propio sector de la economía social de cada país, y en segundo lugar, conocer el grado de conocimiento y notoriedad de otros conceptos afines, en particular, las 'empresas sociales', el 'sector no lucrativo', el 'tercer sector' y las 'cooperativas'. 
La metodología utilizada para este objetivo es análoga a la del primer capítulo del estudio de CIRIEC The Enterprises and Organizations of the Third System. A Strategic Challenge for Employment (Vivet y Thiry, 2000). La percepción de la visibilidad de estos conceptos se ha realizado por expertos y testigos privilegiados de esta realidad social, en concreto por académicos y profesionales de las estructuras federativas y representativas de este sector de sus respectivos países. En el caso del estudio de los países europeos (Monzón y Chaves, 2012), el acopio de información se realizó mediante un cuestionario con preguntas semi-cerradas, mientras para el estudio de los países iberoamericanos y del norte de África (Radrigán, 2012), la valoración se realizó únicamente por académicos expertos. El grado de reconocimiento se ha modulado de modo relativo (nunca absoluto) por número de estrellas: (*) cuando existe un nivel de reconocimiento escaso o nulo de ese concepto en el país; (**) cuando existe un nivel moderado de reconocimiento; y (***) cuando existe un nivel elevado que denota un reconocimiento institucionalizado del concepto en el país en cuestión. Los resultados se presentan en los cuadros 1 a 6 que reflejan respectivamente el nivel de reconocimiento del concepto (y el término) de economía social y el reconocimiento de los conceptos afines que se acaban de citar.

Cuadro 1. Europa: aceptación nacional del concepto «Economía Social»

\begin{tabular}{|c|c|c|c|}
\hline & $\begin{array}{c}\text { Por las autoridades } \\
\text { públicas }\end{array}$ & $\begin{array}{l}\text { Por las empresas de } \\
\text { la economía social }\end{array}$ & $\begin{array}{c}\text { Por el mundo } \\
\text { académico - científico }\end{array}$ \\
\hline Austria & $*$ & $* *$ & $* *$ \\
\hline Bélgica & $* *$ & $* * *$ & $* *$ \\
\hline Dinamarca & $* *$ & $* *$ & $* *$ \\
\hline Finlandia & $* *$ & $* *$ & $* *$ \\
\hline Francia & $* *$ & $* * *$ & $* *$ \\
\hline Alemania & $*$ & $* *$ & $* *$ \\
\hline Grecia & $* *$ & $* *$ & $* * *$ \\
\hline Irlanda & $* *$ & $* * *$ & $* *$ \\
\hline Italia & $* *$ & $* *$ & $* *$ \\
\hline Luxemburgo & $* *$ & $* *$ & $* *$ \\
\hline Portugal & $* * *$ & $* * *$ & $* *$ \\
\hline Países Bajos & $*$ & $*$ & $*$ \\
\hline España & $* * *$ & $* * *$ & $* * *$ \\
\hline Suecia & $* *$ & $* *$ & $*$ \\
\hline Reino Unido & $*$ & $* *$ & $* *$ \\
\hline Bulgaria & $* *$ & $* *$ & $* *$ \\
\hline Chipre & $* *$ & $* *$ & $* *$ \\
\hline República Checa & $*$ & $* *$ & $* *$ \\
\hline Estonia & $* *$ & $*$ & $*$ \\
\hline Hungría & $*$ & $* *$ & $*$ \\
\hline Letonia & $*$ & $* *$ & $* *$ \\
\hline
\end{tabular}

REVESCO No 112 - Extraordinario en Homenaje al Profesor Alfonso Carlos MORALES

GUTIÉRREZ - ISSN: 1885-8031 - www.ucm.es/info/revesco 


\begin{tabular}{|c|c|c|c|}
\hline Lituania & $* *$ & $*$ & $*$ \\
\hline Malta & $* *$ & $*$ & $* *$ \\
\hline Polonia & $* *$ & $* *$ & $* *$ \\
\hline Rumanía & $*$ & $*$ & $*$ \\
\hline Eslovaquia & $*$ & $*$ & $*$ \\
\hline Eslovenia & $*$ & $* *$ & $* *$ \\
\hline Croacia & $*$ & $*$ & $*$ \\
\hline Islandia ** & $* *$ & $* *$ & $* *$ \\
\hline
\end{tabular}

** Países candidatos.

Fuente: Monzón y Chaves, dir, (2012).

Cuadro 2. Iberoamérica: aceptación nacional del concepto «Economía Social»

\begin{tabular}{|l|c|c|c|}
\hline \multicolumn{1}{|c|}{ País } & Por las Autoridades Públicas & $\begin{array}{c}\text { Por las Empresas de } \\
\text { Economía Social }\end{array}$ & $\begin{array}{c}\text { Por el Mundo } \\
\text { Académico Científico }\end{array}$ \\
\hline Argentina & $* * *$ & $* * *$ & $* *$ \\
\hline Bolivia & $*$ & $*$ & $* *$ \\
\hline Brasil & $*$ & $*$ & $*$ \\
\hline Chile & $*$ & $* * *$ & $* *$ \\
\hline Colombia & $* * *$ & $*$ & $* *$ \\
\hline Costa Rica & $*$ & $*$ & $*$ \\
\hline Cuba & $*$ & $*$ & $*$ \\
\hline Ecuador & $*$ & $*$ & $*$ \\
\hline El Salvador & $*$ & $*$ & $*$ \\
\hline Guatemala & $*$ & $* *$ & $* *$ \\
\hline Honduras & $* * *$ & $* *$ & $*$ \\
\hline México & $* *$ & $*$ & $*$ \\
\hline Nicaragua & $*$ & $*$ & $*$ \\
\hline Paraguay & $*$ & $*$ & $*$ \\
\hline Perú & $*$ & $*$ & $*$ \\
\hline Uruguay & $*$ & $*$ & $*$ \\
\hline Venezuela & $*$ & $*$ & $*$ \\
\hline
\end{tabular}

Fuente: Radrigán, coord. (2012).

Cuadro 3. Países del Norte de África: aceptación nacional del concepto «Economía Social»

\begin{tabular}{|l|c|c|c|}
\hline \multicolumn{1}{|c|}{ País } & Por las Autoridades Públicas & $\begin{array}{c}\text { Por las Empresas de } \\
\text { Economía Social }\end{array}$ & $\begin{array}{c}\text { Por el Mundo } \\
\text { Académico Científico }\end{array}$ \\
\hline Argelia & $*$ & $*$ & $*$ \\
\hline Egipto & $*$ & $*$ & $*$ \\
\hline Marruecos & $* * *$ & $* * *$ & $*$ \\
\hline Túnez & $* * *$ & $* * *$ & $*$ \\
\hline
\end{tabular}

Fuente: Radrigán, coord. (2012).

REVESCO No 112 - Extraordinario en Homenaje al Profesor Alfonso Carlos MORALES

GUTIÉRREZ - ISSN: 1885-8031 - www.ucm.es/info/revesco 
Ha sido posible agrupar los países en tres grupos dependiendo del su nivel de reconocimiento del concepto de economía social, conscientes que en muchos casos las condiciones e ideas nacionales asociadas al término de «economía social» difieran considerablemente y puedan no resultar comparables. Las agrupaciones son las siguientes:

- países en los que el concepto de economía social está ampliamente aceptado: En Europa es en España, Francia, Portugal, Bélgica, Irlanda y Grecia donde el concepto de economía social goza de un mayor reconocimiento por parte de las autoridades públicas y el mundo académico y científico, así como por el propio sector de la economía social. En Iberoamérica es en Argentina, Colombia y Honduras donde la economía social goza de mayor aceptación, mientras Marruecos y Túnez son los países del norte de África donde presenta mayor reputación. En todos estos países, en al menos uno de los tres ámbitos (autoridades, empresas de economía social, académico) el nivel relativo de aceptación del concepto de economía social aparece con la máxima puntuación (***). También debe de ponerse de relieve que es el propio sector de la economía social el ámbito de los tres que más se auto reconoce y acepta bajo esta denominación, claramente por delante de las autoridades públicas y del ámbito académico. Cabe destacar tres países: Francia, cuna de este concepto, España, donde se aprobó la primera ley nacional europea de economía social en 2011 y Colombia, primer país americano en aprobar una ley de economía solidaria.

- países en los que existe un reconocimiento escaso o nulo del concepto de economía social: En Europa el concepto de economía social es poco conocido, incipiente o desconocido, entendiendo por ello cuando en dos o tres de los ámbitos contemplados el grado de aceptación es mínimo $\left(^{*}\right)$, en los siguientes países: Estonia, Hungría, Lituania, los Países Bajos, Eslovaquia, Rumania y Croacia. Se trata en su mayor parte de países que se adhirieron a la Unión Europea en sus últimas ampliaciones y por tanto donde no han calado lo suficiente los efectos de difusión y estructurantes de las políticas de las instituciones europeas (ver Demoustier et al, 2006). En contraste, los conceptos afines «sector no lucrativo» y «ONGorganización no gubernamental» sí gozan de una gran aceptación social. En Iberoamérica y norte de África son la gran mayoría los países donde el concepto de economía social carece aún de aceptación social o ésta es muy escasa. En contraposición, en los países iberoamericanos se constata una amplia aceptación social del concepto de 'cooperativas' mientras en los países del norte de África gozan de reputación los conceptos de ONG y de sector no lucrativo. Sólo Méjico (y posiblemente Venezuela, donde sin embargo la locución 
de economía social parece presentar otra acepción) se desmarca presentando unos grados intermedios de aceptación social del concepto de economía social. Llama la atención la escasa aceptación en este país centroamericano de los conceptos de 'cooperativas' (posiblemente una mala imagen), de 'sector no lucrativo' y de 'tercer sector' y en cambio el alto nivel de reputación de las 'empresas sociales y asociativas'.

- países en los que el concepto de economía social goza de un nivel moderado de aceptación: Salvo el caso mejicano antes citado, son solo países europeos, como Italia, Alemania, Reino Unido, Chipre, Dinamarca, Finlandia, Luxemburgo, Suecia, Letonia, Malta, Polonia, Bulgaria e Islandia donde el concepto de economía social presenta una aceptación social moderada, coexistiendo generalmente con otros conceptos afines como el de sector no lucrativo, tercer sector, sector voluntario y empresas sociales, conceptos que alcanzan en algunos países a ser predominantes. En buena parte de los nuevos países miembros de la Unión (Chequia, Hungría, Letonia, Eslovaquia, Croacia y Austria) y el país candidato Islandia, el concepto de 'Nonprofit sector' es el predominante, mientras en países como Alemania, Reino Unido, Polonia, Suecia, Países Bajos y Finlandia son los conceptos de 'Tercer sector' y de 'Empresas sociales' los más aceptados socialmente. Destacar que Finlandia cuenta con la más antigua Ley de empresas sociales de Europa que data del año 2003, muy anterior a la primera ley de economía social.

Cuadro 4. Europa: aceptación nacional de otros conceptos reconocidos relacionados con la economía social

\begin{tabular}{|l|c|c|c|}
\hline & $\begin{array}{c}\text { Empresas } \\
\text { sociales }\end{array}$ & $\begin{array}{c}\text { Sector No } \\
\text { Lucrativo }\end{array}$ & Tercer Sector \\
\hline Austria & $* *$ & $* * *$ & $*$ \\
\hline Bélgica & $* *$ & $* *$ & $*$ \\
\hline Dinamarca & $* *$ & $* * *$ & $* * *$ \\
\hline Finlandia & $* * *$ & $* *$ & $* * *$ \\
\hline Francia & $* *$ & $* *$ & $* *$ \\
\hline Alemania & $* *$ & $* *$ & $* * *$ \\
\hline Grecia & $* *$ & $* *$ & $*$ \\
\hline Irlanda & $* *$ & $* * *$ & $* *$ \\
\hline Italia & $* *$ & $* * *$ & $* *$ \\
\hline Luxemburgo & $*$ & $*$ & $* *$ \\
\hline Portugal & $* *$ & $* *$ & $*$ \\
\hline Países Bajos & $* * *$ & $* * *$ & $*$ \\
\hline España & $*$ & $*$ & $* *$ \\
\hline Suecia & $* * *$ & $* *$ & $* *$ \\
\hline Reino Unido & $* *$ & $* *$ & $* *$ \\
\hline Bulgaria & $* *$ & $* *$ & $*$ \\
\hline
\end{tabular}

REVESCO No 112 - Extraordinario en Homenaje al Profesor Alfonso Carlos MORALES

GUTIÉRREZ - ISSN: 1885-8031 - www.ucm.es/info/revesco 


\begin{tabular}{|l|c|c|c|}
\hline Chipre & $* *$ & $* *$ & $* *$ \\
\hline República Checa & $*$ & $* * *$ & $* *$ \\
\hline Estonia & $*$ & $* *$ & $* *$ \\
\hline Hungría & $*$ & $* * *$ & $*$ \\
\hline Letonia & $*$ & $* * *$ & $* *$ \\
\hline Lituania & $*$ & $* *$ & $* *$ \\
\hline Malta & $* *$ & $* *$ & $*$ \\
\hline Polonia & $* * *$ & $* *$ & $* * *$ \\
\hline Rumania & $*$ & $* *$ & $*$ \\
\hline Eslovaquia & $* *$ & $* * *$ & $* * *$ \\
\hline Eslovenia & $*$ & $* *$ & $*$ \\
\hline Croacia & $*$ & $* * *$ & $* * *$ \\
\hline Islandia $* *$ & $* *$ & $* * *$ & $* *$ \\
\hline
\end{tabular}

** Países candidatos.

Fuente: Monzón y Chaves, dir, (2012).

En los países europeos francófonos (Francia, la región valona de Bélgica y Luxemburgo), los conceptos de «economía solidaria» y «economía social y solidaria» también se reconocen, mientras que la idea de Gemeinwirtschaft (economía de interés general) se conoce en países germánicos como Alemania y Austria. Es importante señalar que, en varios países, no se reconocen determinados componentes del término «economía social» en su sentido más amplio como parte integrante de este sector, haciendo hincapié por el contrario en su especificidad y distanciamiento. Este es el caso de las cooperativas en países como Alemania (Munkner, 1993; Monzón y Chaves, 2012).

Cuadro 5. Iberoamérica: aceptación nacional de otros conceptos reconocidos relacionados con la economía social

\begin{tabular}{|l|c|c|c|c|}
\hline \multicolumn{1}{|c|}{ País } & Cooperativas & $\begin{array}{c}\text { Sector No Lucrativo } \\
\text { (Asociaciones - ONG) }\end{array}$ & Tercer Sector & $\begin{array}{c}\text { Empresas Sociales } \\
\text { - Asociativas }\end{array}$ \\
\hline Argentina & $* * *$ & $* * *$ & $*$ & $*$ \\
\hline Bolivia & $* * *$ & $*$ & $* * *$ & $*$ \\
\hline Brasil & $* * *$ & $* * *$ & $* *$ & $*$ \\
\hline Chile & $* * *$ & $* * *$ & $*$ & $*$ \\
\hline Colombia & $* * *$ & $* * *$ & $*$ & $*$ \\
\hline Costa Rica & $* * *$ & $* * *$ & $*$ & $*$ \\
\hline Cuba & $* * *$ & $*$ & $*$ & $*$ \\
\hline Ecuador & $* * *$ & $*$ & $*$ & $*$ \\
\hline El Salvador & $* * *$ & $*$ & $*$ & $*$ \\
\hline Guatemala & $* * *$ & $*$ & $* *$ \\
\hline Honduras & $* * *$ & $*$ & $*$ & $*$ \\
\hline México & $* *$ & $* *$ & $*$ \\
\hline
\end{tabular}

REVESCO No 112 - Extraordinario en Homenaje al Profesor Alfonso Carlos MORALES

GUTIÉRREZ - ISSN: 1885-8031 - www.ucm.es/info/revesco 


\begin{tabular}{|l|c|c|c|c|}
\hline Nicaragua & $* * *$ & $*$ & $*$ & $*$ \\
\hline Paraguay & $* * *$ & $*$ & $*$ & $*$ \\
\hline Perú & $* * *$ & $*$ & $*$ & $* *$ \\
\hline Uruguay & $* * *$ & $*$ & $*$ & $*$ \\
\hline Venezuela & $* * *$ & $*$ & $* *$ \\
\hline
\end{tabular}

Fuente: Radrigán, coord. (2012).

Cuadro 6. Norte de Africa: aceptación nacional de otros conceptos reconocidos relacionados con la economía social

\begin{tabular}{|l|c|c|c|c|}
\hline \multicolumn{1}{|c|}{ País } & Cooperativas & $\begin{array}{c}\text { Sector No Lucrativo } \\
\text { (Asociaciones - ONG) }\end{array}$ & Tercer Sector & $\begin{array}{c}\text { Empresas Sociales } \\
\text { - Asociativas }\end{array}$ \\
\hline Argelia & $* * *$ & $* * *$ & $*$ & $*$ \\
\hline Egipto & $* * *$ & $* * *$ & $*$ & $*$ \\
\hline Marruecos & $* * *$ & $* * *$ & $*$ & $*$ \\
\hline Túnez & $* * *$ & $* * *$ & $*$ & $*$ \\
\hline
\end{tabular}

Fuente: Radrigán, coord. (2012).

En conclusión, de los resultados anteriores puede constatarse que nos hallamos aún lejos de un consenso internacional e incluso nacional en cuanto a la aceptación de una única concepción del campo que abarca 'lo que no es sector público ni privado tradicional capitalista'. En muchos países la concepción de 'economía social' ha adquirido una elevada notoriedad y reconocimiento, sin embargo, son muchos más aquellos países donde esta concepción coexiste con otras, las cuales en varios casos alcanzan mayores niveles de aceptación que la primera. Finalmente, pero no por ello menos importante, indicar que en todos los países, dada su larga tradición histórica, las cooperativas gozan de un amplio reconocimiento social. En gran medida esta situación también se da para las 'ONG'.

\section{EL RETO DEL RECONOCIMIENTO INSTITUCIONAL}

La forma canónica de cristalizar el reconocimiento institucional de una realidad socioeconómica es a través de su consideración en la normativa, recogiéndola en las políticas públicas generales y específicas, estableciendo órganos públicos especializados dedicados a esta realidad económica y sobre todo aprobando leyes que la regulan.

Desde la pasada década han sido varios los gobiernos europeos e iberoamericanos que han prestado atención a la economía social, llegando, en algunos casos, a promulgar leyes de economía social y/o de empresas sociales. Y es en los procesos de discusión de estas leyes 
cuando se han avivado de nuevo (anteriormente lo fue en el ámbito académico) los debates conceptuales sobre este 'tercer sector' y su alcance sociopolítico en el país. En algún caso, como el del proyecto de ley de economía solidaria francés del gobierno Jospin hace diez años, las discusiones y falta de consenso acabaron haciendo encallar este proyecto; en otros, como el caso mejicano, sufrieron enconados enfrentamientos y reveses, con sucesivos textos globales. El propio texto de la ley española experimentó profundos cambios en el proceso legislativo desde el proyecto inicial propuesto por el grupo de expertos (Monzón, 2009).

El cuadro 7 recoge el panorama actual del reconocimiento jurídico en leyes específicas de la economía social o de las empresas sociales en Europa e Iberoamérica. En Europa son ya cinco las leyes de economía social aprobadas, tres nacionales (España, Grecia y Portugal) y tres regionales (Valonia, Bruselas y Flandes en Bélgica). En Iberoamérica, tras la decana Ley de economía solidaria colombiana, se han aprobado en Ecuador y en Méjico sendas leyes con denominaciones próximas. Francia, si bien aún no cuenta con Ley de economía social, dispone en la actualidad de un proyecto de ley de economía social y solidaria en proceso parlamentario y por primera vez en la historia de un Ministerio para la economía social y solidaria.

Cuadro 7. Reconocimiento jurídico de la economía social o del estatuto de empresa social en Europa e Iberoamérica

\begin{tabular}{|l|l|l|}
\hline \multicolumn{1}{|c|}{ País } & \multicolumn{1}{c|}{ Ley } & \multicolumn{1}{c|}{ Nombre de la Ley/ Proyecto de ley } \\
\hline España & SI & Economía Social (2011) \\
\hline Grecia & SI & Economía Social y empresas sociales (2011) \\
\hline Bélgica (Valonia, Bruselas, Flandes) & SI & Economía Social (2008, 2012, Décret régional) \\
\hline Portugal & SI & Economía Social (2013) \\
\hline Finlandia & SI & Empresas sociales (2003) \\
\hline Lituania & SI & Empresas sociales (2004) \\
\hline Italia & SI & Empresas sociales (2005) \\
\hline Eslovenia & SI & Empresas sociales (2011) \\
\hline Colombia & SI & Economía solidaria (1998) \\
\hline Ecuador & SI & Economía popular y solidaria (2011) \\
\hline México & SI & Economía social y solidaria (2012) \\
\hline Francia & Proyecto & Economía Social (2013) \\
\hline Polonia & Proyecto & Economía Social (2012) \\
\hline Países Bajos & Proyecto & Empresas sociales (2012) \\
\hline Rumania & Proyecto & Empresas sociales (2012) \\
\hline
\end{tabular}


Las tres leyes nacionales europeas de economía social vigentes en la actualidad se encuentran en sendos países que están sufriendo la crisis con mayor dureza: España, Grecia y Portugal. La primera es la Ley española 5/2011 de 29 de marzo de 2011 de Economía Social, la segunda es la Ley griega 4019/2011 de Economía Social, Emprendimiento Social y otras disposiciones y la tercera es la Ley 30/2013 de 8 de mayo, de Bases da Economía Social de Portugal.

Es interesante constatar cómo coinciden estos países con los países donde el concepto de economía social goza de mayor aceptación social, como se ha mostrado en el apartado precedente.

\section{EL RETO DE LA CUANTIFICACIÓN}

Conocer, con estadísticas rigurosas, las dimensiones cuantitativas de la economía social constituye otro de los principales retos. Es conocido el dicho 'lo que no se cuenta (y/o lo que representa muy poco) no importa ni cuenta para las decisiones importantes', dicho refrendado en la teoría política de Kingdom (1984), en concreto en el concepto de 'apertura de la ventana política' a un problema o realidad al objeto de que éste sea integrado en la agenda política y consiguientemente en las políticas públicas.

Han sido escasos, hasta el presente, los estudios de carácter nacional o internacional que han ofrecido estadísticas comparadas sobre la economía social en los países de los tres continentes que se abordan en este artículo. Deben de destacarse para Europa, los estudios de CIRIEC para la Comisión Europea y para el Comité Económico y Social Europeo, para el Norte de África los estudios de CIRIEC-España y para Iberoamérica los estudios de FUNDIBES, la Fundación iberoamericana para la economía social. El panorama revela situaciones muy desiguales entre los diferentes países, con amplias diferencias en disponibilidad de información, rigurosidad y por tanto de fiabilidad de los datos, así como sistematización en su acopio. En los últimos años diversos institutos nacionales de estadística de Europa e Iberoamérica se han esforzado por proporcionar datos fidedignos sobre varios grupos de economía social. La propia Comisión Europea ha elaborado un Manual de Cuentas Satélite para Cooperativas y Mutualidades (Monzón y Barea, 2007), complementario al Handbook de las nonprofit de Naciones Unidas (United Nations, 2003). También se han desarrollado metodologías de evaluación de la economía social (Bouchard, 2010). En España, Portugal, la República Checa y Hungría se ha utilizado la metodología de las cuentas satélite. 
Otros institutos de estadística, como el de Francia, proporcionan series temporales sobre el empleo en la economía social. No obstante, queda mucho por hacer, y durante los próximos años deberán realizarse grandes esfuerzos para sistematizar las estadísticas.

A continuación se presentan los resultados de sendos trabajos de referencia para el presente artículo (Monzón y Chaves, 2012) y Radrigán (2012). Se ofrece una visión general de las cifras claves de la economía social por país, divididos por continentes, a nivel global y diferenciando tres grupos de organizaciones: cooperativas y formas similares aceptadas; mutuas y formas similares; y por último, asociaciones, fundaciones y otras formas afines sin ánimo de lucro. Las cifras estudiadas son el número de personas empleadas, el número de miembros y el número de entidades o empresas. La información estadística recabada ha procedido de fuentes secundarias, tomando como periodo de referencia, en lo posible, el bienio 2009-2010. Es especialmente elocuente la laguna estadística en numerosos países iberoamericanos y del norte de África, pero también de múltiples países europeos, sobre todo de los nuevos países miembros.

Teniendo en cuenta la metodología utilizada, particularmente en vista de la dificultad para comparar determinadas variables a nivel internacional, la dudosa fiabilidad de los datos sobre algunos países, el riesgo de doble contabilidad dentro de las «familias» de un único país, a la disponibilidad o no de datos, esta información estadística debe tratarse con prudencia.

En cualquier caso, se trata de una información cuantitativa que por primera vez se pone en comparación internacional con tres continentes de referencia y se presenta su peso relativo en la sociedad de cada país con los indicadores de tasa de penetración societaria de la economía social (porcentaje de socios en la economía social / total de la población) y de tasa de empleo en la economía social sobre el total del empleo nacional. Los cuadros 8 a 13 ofrecen con detalle los resultados sintéticos obtenidos.

La principal conclusión que se desprende de esta información estadística, a pesar, insistimos, de las importantes lagunas y deficiencias en cuanto a fuentes de información para muchos países, es que la economía social constituye una realidad importante en términos humanos y económicos, aunque también presenta un arraigo muy desigual según países y continentes.

REVESCO No 112 - Extraordinario en Homenaje al Profesor Alfonso Carlos MORALES

GUTIÉRREZ - ISSN: 1885-8031 - www.ucm.es/info/revesco 
En Europa, donde se cuenta con información estadística más amplia y sistematizada, la economía social emplea a más de 14,5 millones de personas que representan cerca del 6,5\% de la población ocupada de la Unión Europea de los 27. En algunos países europeos como Suecia, Bélgica, Italia, Francia y Países Bajos la economía social representa entre el 9 y el $11,5 \%$ del total de la población ocupada. Estas tasas son significativamente inferiores en los nuevos países miembros de la Unión, caracterizados también por menores niveles de renta per cápita, desarrollo económico y bienestar.

En los países iberoamericanos y norte de África la disponibilidad y fiabilidad de la información es menor, aún así se observa un interesante panorama. Resulta obvio indicar que son necesarios serios esfuerzos para construir estadísticas que permitan precisar con mayor claridad las dimensiones sociales y económicas de la economía social en estos países. Debe ponerse de relieve que es el ámbito de las cooperativas el que dispone de una mayor información estadística. Tomando los datos obtenidos con prudencia, la realidad de la economía social en estos continentes del Sur no deja de ser importante cuantitativamente: se computan más de 52 millones de socios de entidades de economía social en Iberoamérica y casi 16 millones en los cuatro países del norte de África, en total casi 70 millones de personas. La tasa de penetración societaria de la economía social alcanza niveles significativos en varios países como Uruguay, Argentina, Bolivia, Costa Rica y Paraguay, con más de un $20 \%$ de la población socia de alguna entidad de economía social y niveles medianos (situados entre el 10 y el 20\%) en países como Ecuador, Egipto, Colombia, Marruecos y Honduras.

Cuadro 8. Europa: Empleos remunerados en las cooperativas, mutuas y asociaciones en la UE, (20092010)

\begin{tabular}{|l|r|r|r|r|}
\hline \multicolumn{1}{|c|}{ País } & Cooperativas & Mutualidades & Asociaciones & \multicolumn{1}{c|}{ TOTAL } \\
\hline Austria & 61.999 & 1.416 & 170.113 & 233.528 \\
\hline Bélgica & 13.547 & 11.974 & 437.020 & 462.541 \\
\hline Dinamarca & 70.757 & 4.072 & 120.657 & 195.486 \\
\hline Finlandia & 94.100 & 8.500 & 84.600 & 187.200 \\
\hline Francia & 320.822 & 128.710 & 1.869 .012 & 2.318 .544 \\
\hline Alemania & 830.258 & 86.497 & 1.541 .829 & 2.458 .584 \\
\hline Grecia & 14.983 & 1.140 & 101.000 & 117.123 \\
\hline Irlanda & 43.328 & 650 & 54.757 & 98.735 \\
\hline Italia & 1.128 .381 & n.a. & 1.099 .629 & 2.228 .010 \\
\hline Luxemburgo & 1.933 & n.a. & 14.181 & 16.114 \\
\hline Portugal & 51.391 & 5.500 & 194.207 & 251.098 \\
\hline Países Bajos & 184.053 & 2.860 & 669.121 & 856.054 \\
\hline
\end{tabular}

REVESCO No 112 - Extraordinario en Homenaje al Profesor Alfonso Carlos MORALES GUTIÉRREZ - ISSN: 1885-8031 - www.ucm.es/info/revesco 


\begin{tabular}{|l|r|r|r|r|} 
España & 646.397 & 8.700 & 588.056 & 1.243 .153 \\
\hline Suecia & 176.816 & 15.825 & 314.568 & 507.209 \\
\hline Reino Unido & 236.000 & 50.000 & 1.347 .000 & 1.633 .000 \\
\hline Bulgaria & 41.300 & n.a. & 80.000 & 121.300 \\
\hline Chipre & 5.067 & n.a. & n.a. & 5.067 \\
\hline República Checa & 58.178 & 5.679 & 96.229 & 160.086 \\
\hline Estonia & 9.850 & n.a. & 28.000 & 37.850 \\
\hline Hungría & 85.682 & 6.676 & 85.852 & 178.210 \\
\hline Letonia & 440 & n.a. & n.a. & 440 \\
\hline Lituania & 8.971 & n.a. & n.a. & 8.971 \\
\hline Malta & 250 & n.a. & 1.427 & 1.677 \\
\hline Polonia & 400.000 & 2.800 & 190.000 & 592.800 \\
\hline Rumanía & 34.373 & 18.999 & 109.982 & 163.354 \\
\hline Eslovaquia & 26.090 & 2.158 & 16.658 & 44.906 \\
\hline Eslovenia & 3.428 & 476 & 3.190 & 7.094 \\
\hline Croacia & 3.565 & 1.569 & 3.950 & 9.084 \\
\hline Islandia ** & n.a. & 221 & n.a. & 221 \\
\hline TOTAL EU-15 & $\mathbf{3 . 8 7 4 . 7 6 5}$ & $\mathbf{3 2 5 . 8 4 4}$ & $\mathbf{8 . 6 0 5 . 7 5 0}$ & $\mathbf{1 2 . 8 0 6 . 3 7 9}$ \\
\hline $\begin{array}{l}\text { Nuevos estados } \\
\text { miembros (12) }\end{array}$ & $\mathbf{6 7 3 . 6 2 9}$ & $\mathbf{3 6 . 7 8 8}$ & $\mathbf{6 1 1 . 3 3 8}$ & $\mathbf{1 . 3 2 1 . 7 5 5}$ \\
\hline TOTAL EU-27 & $\mathbf{4 . 5 4 8 . 3 9 4}$ & $\mathbf{3 6 2 . 6 3 2}$ & $\mathbf{9 . 2 1 7 . 0 8 8}$ & $\mathbf{1 4 . 1 2 8 . 1 3 4}$ \\
\hline
\end{tabular}

Nota: ** Países candidatos; En Italia, los datos de mutuas y cooperativas se han agregado. Fuente: Monzón y Chaves, dir, (2012).

Cuadro 9. Europa: Empleos remunerados en la economía social en comparación con el empleo remunerado total en la UE (2009-2010), en miles

\begin{tabular}{|l|r|r|r|}
\hline \multicolumn{1}{|c|}{ País } & $\begin{array}{r}\text { Empleo en la } \\
\text { ES }\end{array}$ & \multicolumn{1}{c|}{$\begin{array}{c}\text { Total } \\
\text { Empleo }\end{array}$} & \multicolumn{1}{c|}{$\%$} \\
\hline Austria & 233,53 & $4.096,30$ & $5,70 \%$ \\
\hline Bélgica & 462,54 & $4.488,70$ & $10,30 \%$ \\
\hline Dinamarca & 195,49 & $2.706,10$ & $7,22 \%$ \\
\hline Finlandia & 187,2 & $2.447,50$ & $7,65 \%$ \\
\hline Francia & $2.318,54$ & $25.692,30$ & $9,02 \%$ \\
\hline Alemania & $2.458,58$ & $38.737,80$ & $6,35 \%$ \\
\hline Grecia & 117,12 & $4.388,60$ & $2,67 \%$ \\
\hline Ireland & 98,74 & $1.847,80$ & $5,34 \%$ \\
\hline Italia & $2.228,01$ & $22.872,30$ & $9,74 \%$ \\
\hline Luxemburgo & 16,11 & 220,8 & $7,30 \%$ \\
\hline Portugal & 251,1 & $4.978,20$ & $5,04 \%$ \\
\hline Países Bajos & 856,05 & $8.370,20$ & $10,23 \%$ \\
\hline España & $1.243,15$ & $18.456,50$ & $6,74 \%$ \\
\hline Suecia & 507,21 & $4.545,80$ & $11,16 \%$ \\
\hline Reino Unido & $1.633,00$ & $28.941,50$ & $5,64 \%$ \\
\hline Bulgaria & 121,3 & $3.052,80$ & $3,97 \%$ \\
\hline Chipre & 5,07 & 385,1 & $1,32 \%$ \\
\hline
\end{tabular}

REVESCO No 112 - Extraordinario en Homenaje al Profesor Alfonso Carlos MORALES 


\begin{tabular}{|l|r|r|r|} 
República Checa & 160,09 & $4.885,20$ & $3,28 \%$ \\
\hline Estonia & 37,85 & 570,9 & $6,63 \%$ \\
\hline Hungría & 178,21 & $3.781,20$ & $4,71 \%$ \\
\hline Letonia & 0,44 & 940,9 & $0,05 \%$ \\
\hline Lituania & 8,97 & $1.343,70$ & $0,67 \%$ \\
\hline Malta & 1,68 & 164,2 & $1,02 \%$ \\
\hline Polonia & 592,8 & $15.960,50$ & $3,71 \%$ \\
\hline Rumanía & 163,35 & $9.239,40$ & $1,77 \%$ \\
\hline Eslovaquia & 44,91 & $2.317,50$ & $1,94 \%$ \\
\hline Eslovenia & 7,09 & 966 & $0,73 \%$ \\
\hline Croacia & 9,08 & $1.541,20$ & $0,59 \%$ \\
\hline Islandia ** & 0,22 & 165,8 & $0,13 \%$ \\
\hline TOTAL EU-15 & $12.806,37$ & $172.790,40$ & $7,41 \%$ \\
\hline TOTAL EU-27 & $14.128,13$ & $\mathbf{2 1 6 . 3 9 7 , 8 0}$ & $6,53 \%$ \\
\hline
\end{tabular}

Nota: ** Países candidatos; * Población ocupada entre 16 y 65 años, Eurostat, 2010. Fuente: Monzón y Chaves, dir, (2012).

Cuadro 10. Iberoamérica: Socios de las entidades de economía social y número de empleos remunerados en las cooperativas, 2005-10 según país.

\begin{tabular}{|l|r|r|r|r|r|r|}
\hline \multicolumn{1}{|c|}{ País } & $\begin{array}{c}\text { Empleos } \\
\text { Cooperat. }\end{array}$ & $\begin{array}{c}\text { Socios } \\
\text { Cooperativas }\end{array}$ & $\begin{array}{c}\text { Socios } \\
\text { Mutuales }\end{array}$ & $\begin{array}{c}\text { Socios } \\
\text { Asociaciones }\end{array}$ & $\begin{array}{c}\text { Socios en } \\
\text { otras EES }\end{array}$ & \multicolumn{1}{c|}{ Total Socios } \\
\hline Argentina & 289.460 & 9.392 .713 & 4.997 .067 & - & - & 14.389 .780 \\
\hline Bolivia & 32.323 & 3.000 .000 & - & - & - & 3.000 .000 \\
\hline Brasil & 251.000 & 7.690 .000 & - & - & 1.681 .496 & 9.371 .496 \\
\hline Chile & 36.679 & 1.178 .688 & 40.000 & - & - & 1.218 .688 \\
\hline Colombia & 139.703 & 5.131 .780 & 206.740 & - & - & 5.338 .520 \\
\hline Costa Rica & 59.380 & 777.713 & - & 425.000 & - & 1.202 .713 \\
\hline Cuba & - & & - & - & - & 0 \\
\hline Ecuador & - & 1.808 .788 & - & - & - & 1.808 .788 \\
\hline El Salvador & - & 281.352 & - & - & 183.204 & 464.556 \\
\hline Guatemala & - & 1.225 .359 & - & - & - & 1.225 .359 \\
\hline Honduras & - & 7.000 & - & - & 805.000 & 812.000 \\
\hline México & 4.864 .448 & 3.051 .281 & - & - & 4.948 .720 & 8.000 .001 \\
\hline Nicaragua & - & & - & - & - & 0 \\
\hline Paraguay & - & 1.292 .671 & - & - & 2.862 & 1.295 .533 \\
\hline Perú & - & 775.391 & - & - & - & 775.391 \\
\hline Uruguay & 1.200 & 800.000 & 700.000 & 400.000 & - & 1.900 .000 \\
\hline Venezuela & - & 1.602 .343 & - & - & 400.778 & 2.003 .121 \\
\hline Total & $\mathbf{5 . 6 7 4 . 1 9 3}$ & $\mathbf{3 8 . 0 1 5 . 0 7 9}$ & $\mathbf{5 . 9 4 3 . 8 0 7}$ & $\mathbf{8 2 5 . 0 0 0}$ & $\mathbf{8 . 0 2 2 . 0 6 0}$ & $\mathbf{5 2 . 8 0 5 . 9 4 6}$ \\
\hline
\end{tabular}

Fuente: Radrigán, coord. (2012). 
Cuadro 11. Norte de África: Socios de las entidades de economía social y número de empleos remunerados en las cooperativas, 2005-10 según país.

\begin{tabular}{|l|r|r|r|r|r|r|}
\hline \multicolumn{1}{|c|}{ País } & $\begin{array}{c}\text { Empleos } \\
\text { Cooperat. }\end{array}$ & $\begin{array}{c}\text { Socios } \\
\text { Cooperativas }\end{array}$ & $\begin{array}{c}\text { Socios } \\
\text { Mutuales }\end{array}$ & $\begin{array}{c}\text { Socios } \\
\text { Asociaciones }\end{array}$ & $\begin{array}{c}\text { Socios en } \\
\text { otras EES }\end{array}$ & \multicolumn{1}{c|}{ Total Socios } \\
\hline Argelia & 20.000 & 286.000 & 1.137 .267 & - & - & 1.423 .267 \\
\hline Egipto & - & 10.148 .000 & - & - & - & 10.148 .000 \\
\hline Marruecos & 22.502 & 365.255 & 3.090 .710 & - & - & 3.455 .965 \\
\hline Túnez & 135.000 & 37.923 & 860.000 & - & - & 897.923 \\
\hline Total & $\mathbf{1 7 7 . 5 0 2}$ & $\mathbf{1 0 . 8 3 7 . 1 7 8}$ & $\mathbf{5 . 0 8 7 . 9 7 7}$ & sd & s/d & $\mathbf{1 5 . 9 2 5 . 1 5 5}$ \\
\hline
\end{tabular}

Fuente: Radrigán, coord. (2012).

Cuadro 12. Iberoamérica: Socios y empleos remunerados en la economía social en comparación con el total del empleo y de la población del país, 2005-10 según país.

\begin{tabular}{|c|c|c|c|c|}
\hline País & $\begin{array}{c}\text { Empleos en } \\
\text { ES } \\
\end{array}$ & Socios en ES & $\begin{array}{c}\text { \% Empleo en ES } \\
\text { / total empleo }\end{array}$ & $\begin{array}{c}\% \text { Socios en ES / } \\
\text { total población }\end{array}$ \\
\hline Argentina & 289.460 & 14.389 .780 & 1,8 & 35,5 \\
\hline Bolivia & 32.323 & 3.000 .000 & 0,7 & 29,0 \\
\hline Brasil & 1.792 .290 & 9.371 .496 & 1,8 & 4,0 \\
\hline Chile & 340.562 & 1.218 .688 & 4,6 & 7,4 \\
\hline Colombia & 144.461 & 5.338 .520 & 0,7 & 11,7 \\
\hline Costa Rica & 100.628 & 1.202 .713 & 5,2 & 26,0 \\
\hline Cuba & - & - & $\mathrm{Nd}$ & $\mathrm{Nd}$ \\
\hline Ecuador & - & 1.808 .788 & $\mathrm{Nd}$ & 12,6 \\
\hline El Salvador & & 464.556 & $\mathrm{Nd}$ & 7,5 \\
\hline Guatemala & - & 1.225 .359 & $\mathrm{Nd}$ & 8,3 \\
\hline Honduras & - & 812.000 & $\mathrm{Nd}$ & 10,1 \\
\hline México & 4.864 .448 & 8.000 .001 & 11,0 & 7,1 \\
\hline Nicaragua & - & - & $\mathrm{Nd}$ & $\mathrm{Nd}$ \\
\hline Paraguay & - & 1.295 .533 & $\mathrm{Nd}$ & 20,3 \\
\hline Perú & 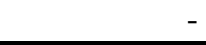 & 775.391 & $\mathrm{Nd}$ & 2,6 \\
\hline Uruguay & 25.200 & 1.900 .000 & 4,3 & 55,5 \\
\hline Venezuela & & 2.003 .121 & $\mathrm{Nd}$ & 6,6 \\
\hline Total & 7.589.372 & 52.805 .946 & & \\
\hline
\end{tabular}

Fuente: Radrigán, coord. (2012). 
Cuadro 13. Norte de África: Socios y empleos remunerados en la economía social en comparación con el total del empleo y de la población del país, 2005-10 según país.

\begin{tabular}{|c|c|c|c|c|}
\hline País & $\begin{array}{c}\text { Empleos en } \\
\text { ES } \\
\end{array}$ & Socios en ES & $\begin{array}{c}\text { \% Empleo en ES } \\
\text { / total empleo }\end{array}$ & $\begin{array}{c}\text { \% Socios en ES / } \\
\text { total población }\end{array}$ \\
\hline Argelia & 23.696 & 1.423 .267 & 0,3 & 4,1 \\
\hline Egipto & & 10.148 .000 & $\mathrm{Nd}$ & 12,2 \\
\hline Marruecos & 22.502 & 3.455 .965 & 0,2 & 11,0 \\
\hline Túnez & 265.365 & 897.923 & 7,0 & 8,8 \\
\hline Total & 311.563 & 15.925.155 & & \\
\hline
\end{tabular}

Fuente: Radrigán, coord. (2012).

\section{CONCLUSIONES}

Ha sido en los últimos años cuando el concepto de economía social se ha consolidado institucionalmente en forma de reconocimiento jurídico específico con leyes de economía social aprobadas o en proyecto en diversos países de Europa e Iberoamérica. Esta institucionalización cristaliza una larga trayectoria de actividad en pro de su conocimiento, visibilidad y fomento, realizada tanto desde el ámbito académico como desde el propio movimiento de la economía social mediante su vertebración y autoreconocimiento.

No obstante, tanto la aceptación social del concepto de economía social y de sus conceptos afines (sector no lucrativo, tercer sector y empresas sociales), como su reconocimiento jurídico y como su cuantificación con estadísticas fiables presentan fuertes disparidades según los países de los tres continentes analizados, Europa, Iberoamérica y norte de África. En todo caso se aprecia una elevada correlación entre una mayor aceptación social del concepto, un mayor reconocimiento jurídico y una mayor disponibilidad y fiabilidad de información estadística así como mayor dimensión cuantitativa de la economía social.

En perspectiva comparada, un grupo de países sobresalen por su mayor grado relativo de reconocimiento social, político y académico del concepto de economía social. Son España, Portugal, Francia, Bélgica, Irlanda, Grecia, Colombia, Argentina, Honduras, Marruecos y Túnez. Varios de estos países ya cuentan con leyes específicas de economía social o con proyecto de ley. Presentan generalmente tasas de penetración societaria o de empleo en la economía social sobre el total del empleo nacional muy superiores, de media, al resto de países analizados.

REVESCO No 112 - Extraordinario en Homenaje al Profesor Alfonso Carlos MORALES

GUTIÉRREZ - ISSN: 1885-8031 - www.ucm.es/info/revesco 
En contraste, existe un gran número de países donde el concepto de economía social y sus concepciones afines aún son desconocidos o poco conocidos. En consecuencia con lo anterior, su grado de reconocimiento institucional y jurídico es escaso o nulo y generalmente presentan menores tasas de penetración societaria en el país o tasa de empleo en la economía social sobre el total del empleo nacional. Este grupo de países lo conforman, en Europa, buena parte de los nuevos países miembros de la Unión, en Iberoamérica la mayor parte de los países exceptuados Méjico y Ecuador y los países citados en el párrafo anterior, y finalmente en el norte de África, Argelia y Egipto, aun reconociendo los esfuerzos realizados en los últimos años en el seno de Mercosur.

\section{BIBLIOGRAFÍA}

A.A.V.V. Cuadernos de economia social, nº 16 (2006), En Homenaje al Doctor Arturo Octavio Ravina, Instituto Argentino de Investigaciones de Economia Social y Fundibes.

ALIANZA COOPERATIVA INTERNACIONAL PARA LAS AMÉRICAS (2009). La Legislación cooperativa en los países de México, Centroamérica y el Caribe. ACI Américas. San José, Costa Rica.

ALIANZA COOPERATIVA INTERNACIONAL PARA LAS AMÉRICAS (2009). La Legislación cooperativa en los países andinos, San José de Costa Rica, ACI - Américas.

BAREA, J. (1990). Concepto y agentes de la economía social, CIRIEC-ESPAÑA, revista de Economía Pública, Social y Cooperativa, no 8, p. 109-117.

BAREA, J. y MONZÓN, J.L. (1995). Las cuentas Satélite de la Economía Social en España; una primera aproximación, Valencia: CIRIEC-España editorial.

BAREA, J. y MONZÓN, J.L. (2006). CIRIEC. Manual for Drawing Up the Satellite Accounts on Cooperatives and Mutual Societies. European Commission. Enterprise and Industry Directorate General.

BOUCHARD, M. (2010). CIRIEC (ed). The worth of the Social Economy. An international Perspective. Peter Lang.

BORZAGA, C. Y DEFOURNY, J. (eds) (2001). The emergence of Social Enterprise. London: Routledge.

CARRASCO, I. (dir) (2009). Diccionario de economía social, Madrid: Ecobook editorial.

CECOP (2006). Social enterprises and worker cooperatives: Comparing models of corporate governance and social Inclusion, CECOOP European Seminar, Manchester. 
CEPES y AECID (2008). Economía social en el Magreb. La situación de Marruecos y Túnez. S.d.

CIRIEC (2000). The Enterprises and Organizations of the Third System: A strategic challenge for employment. Liege: CIRIEC- DG V of the European Union, (edición en español: CIRIEC, Economía Social y Empleo en la UE, Madrid: Ministerio de Trabajo y Asuntos Sociales).

COMMISSION OF THE EUROPEAN COMMUNITIES (1997). Promotion Of The Role Of Asociations And Foundations In Europe, Luxembourg, Office of official publication of the European Communities. Com (97) 241 Final.

COMISIÓN EUROPEA (1989). Comunicación al Consejo "Las Empresas de la Economía Social y la realización del Mercado Europeo sin Fronteras".

COMISIÓN DE LAS COMUNIDADES EUROPEAS. Comunicación al Consejo, al Parlamento Europeo, al Comité Económico y Social Europeo y al Comité de las Regiones sobre fomento de las cooperativas en Europa (23 Febrero 2004, Com(2003)18).

CORAGGIO, J.L. (1995). Desarrollo humano, economía popular y educación, Buenos Aires: Instituto de Estudios y Acción Social.

CORAGGIO, J.L. (1997) Alternativas para o desenvolvimiento humano em um mundo globalizado, Revista proposta, $n^{\circ} 72$, p. 30-38.

CHAVES, R. (1999). La Economía Social como enfoque metodológico, como objeto de estudio y como disciplina científica”, Ciriec-España, revista de economía pública, social y cooperativa, $\mathrm{n}^{\mathrm{o}} 33, \mathrm{p} .115-140$

CHAVES, R. y DEMOUSTIER, D. (dir) (2013). The emergence of social economy in public policy. An international perspective, Bruxelles: Peterlang publishers.

CHAVES, R., FECHER, F. y MONZON, J.L. (ed) (2012). Recent trends in Social Economy research, Annals of Public and Cooperative Economics, Vol. 83, $\mathrm{n}^{\mathrm{o}} 3$.

CHAVES, R. y MONZÓN, J.L. (2008). EESC/CIRIEC. The Social Economy in the European Union, European Economic and Social Committee, Bruxelles.

CHAVES, R. y MONZÓN, J.L. (2012). Beyond the crisis: the social economy, prop of a new model of sustainable economic development, Service business - an international journal, $n^{\circ} 6$, p. $5-26$.

CHAVES, R. y PEREZ DE URALDE, J.M. (dir) (2012). La economía social y la cooperación al desarrollo. Una perspectiva internacional, Valencia: Edl Universidad de Valencia.

REVESCO No 112 - Extraordinario en Homenaje al Profesor Alfonso Carlos MORALES

GUTIÉRREZ - ISSN: 1885-8031 - www.ucm.es/info/revesco 
DEFOURNY, J. y MONZÓN, J.L. (eds.) (1992). Économie sociale (entre économie capitaliste et économie publique) / The Third Sector (cooperatives, mutual and nonprofit organizations). Bruxelles : De Boeck Université - CIRIEC.

DEFOURNY, J. y NYSSENS, M. (2011). Approches européennes et américaines de l'entreprise sociale: une perspective comparative, Revue internationale de l'économie sociale, $\mathrm{n}^{\mathrm{o}} 319$.

DEMOUSTIER, D., CHAVES, R., HUNCOVA, M., Lorenz, G. y SPEAR, R. (2006). Débats autour de la notion d'économie sociale en Europe, Revue Internationale de l'économie sociale, $\mathrm{n}^{\circ} 300$, p. 8-18.

DEMOUSTIER, D. (2011). L'économie sociale et solidaire. S'associer pour entreprendre autrement, Paris : Alternatives économiques/Syros.

FAJARDO, G. (2012). El fomento de la economía social en la legislación española, REVESCO, Revista de Estudios Cooperativos, $\mathrm{n}^{\circ}$ 107, p. 58-97.

FONTANEAU, B.; MORAIS, L.; et al. (2010). Social and Solidarity Economy: building a common concept. Reader $2010-1^{\text {st }}$. International Academy of SSE. ITCILO - Turin.

FUNDIBES (2010). Anuario iberoamericano de la economía social, Valencia: Fundibes.

GRIJPSTRA, D. et al. (2011). The role of mutual societies in the 21st century; IP/A/EMPL/ST/2010-004, PE 464.434; European Parlament Directorate General For Internal Policies; Policy Department A: Economic And Scientific Policy.

GUESLIN, A. (1987). L'invention de l'Economie Sociale, Paris: Economica.

HIGHER COUNCIL FOR CO-OPERATION OF FRANCE (2001). Co-operative movements in the European Union, Paris: DIES.

JIMÉNEZ, J. y MORALES, A.C. (2011). Social economy and the fourth sector, base and protagonist of social innovation, CIRIEC-España, revista de economía pública, social y cooperativa, $\mathrm{n}^{\circ} 73$, p. 33-60.

JULIA, J.F. y CHAVES, R. (ed) (2012). Social economy: a responsible people-oriented economy, Special issue, Service Business, an international journal, Vol. 6, $\mathrm{n}^{\circ} 1$.

KINGDON, J. W. (1987). Agendas, alternatives and public policies, Boston, Longman.

LAVILLE, J.L. (1994). L'Économie solidaire. Une perspective internationale, París: Desclée de Brouwer.

LIANZA, S. y CHEDID, F. (orgs) (2012). A economia solidária na América Latina: realidades nacionais e políticas públicas, Rio de Janeiro : Pró Reitoria de Extensão UFRJ. 
MELIAN, A., CAMPOS, V. y SANCHIS, J.R. (2011). Emprendimiento social y empresas de inserción en España, REVESCO, Revista de Estudios Cooperativos, n 106, p. 150-172.

MONZÓN, J.L. (1987). La Economía Social en España, CIRIEC-España, nº 0, p. 19-29.

MONZÓN, J.L. (dir) (2009). Informe para la elaboración de una Ley de fomento de la economía social en España, Madrid: Ministerio de Trabajo e Inmigración.

MONZÓN, J.L. (dir) (2010). Economía Social y su impacto en la generación de empleo. Claves para un desarrollo con equidad en América Latina. Estudios referidos a Argentina, Brasil, Chile, Colombia, España y Uruguay. Madrid : Edl. Fundibes.

MONZÓN, J.L. et al. (dir) (2003). El Tercer sector no lucrativo en el Mediterráneo. La Economía Social de no mercado. I. España, Francia, Grecia, Italia y Portugal, Valencia: CIRIEC-España.

MONZÓN, J.L. y BAREA, J. (2007). Manual de orientación para la elaboración de una cuenta satélite de las empresas de la Economía Social. Brussels: European Commission.

MONZON, J,L. y CHAVES, R. (2012). EESC/CIRIEC. La Economía Social en la Unión Europea. Bruselas: Comité Económico y Social Europeo.

MONZÓN, J.L. y MARCUELLO, C. (2012). Economía Social y Empresas Sociales, CiriecEspaña, revista de economía pública, social y cooperativa, $\mathrm{n}^{\circ} 75$, Monográfico.

MONZÓN, J.L. y CHAVES, R. (2008). The European Social Economy: concept and dimensions of the third sector, Annals of Public and Cooperative Economics, 79, nº 3, p. $549-577$.

MUNKNER, H. (1993). Panorama d'une économie sociale qui ne se reconnaît pas comme telle: le cas de l'Allemagne, RECMA, n 44-45, p. 101-133.

NOYA, A. y CLARENCE, E. (ed) (2007). The Social Economy: Building inclusive economies, Paris: OECD.

NOYA, A. y NATIVEL, C. (ed) (2003). The non profit sector in a changing economy, Paris: OECD.

OIT (2002). Recomendación sobre Promoción de las Cooperativas (Recomendación 193/2002) OIT - Organización Internacional del Trabajo.

PALOMO, R., SANCHIS, J.R. y SOLER, F. (2010). Las entidades financieras de economía social ante la crisis financiera, REVESCO, Revista de Estudios Cooperativos, $\mathrm{n}^{\circ} 100, \mathrm{p}$. 101-133.

PARLEMENT EUROPEEN (2009). Rapport sur l'économie sociale (Informe Toia)Parlement, (2008/2250/INI). 
PEREZ DE URALDE, J.M. (coord) (2001). Economía Social e Iberoamérica: La Construcción de un Espacio Común, Madrid: Fundibes.

PEREZ DE URALDE, J.M. (dir) (2006). La Economía Social en Iberoamericana. Un acercamiento a su realidad Vol. 1, Valencia: Fundibes.

PEREZ DE URALDE, J.M. (coord.) (2011). Reformas legislativas en el derecho social y solidario iberoamericano, Valencia: Ed. Fundación Divina Pastora, Pontificia Universidad Javeriana y Fundación Iberoamericana de la Economía Social (Fundibes).

PEREZ DE URALDE, J.M. Y RADRIGÁN, M. (dir) (2006). La Economía Social en Iberoamericana. Un acercamiento a su realidad Vol. 2. Valencia: Fundibes.

PERRI, 6. y PESTOFF, V.A. (1993). La recherche sur le Third Sector en Europe: directions et perspectives, Revue des Etudes Coopératives, Mutuelles et Associatives, nº 9, p. 50-68.

POWELL, W. (Ed) (1987). The Nonprofit Sector. A Research Handbook. New Haven and London: Yale University Press.

RADRIGÁN, M. (coord) (2008). La economía social en Iberoamérica. Realidad y proyecciones. Una visión comparada, Valencia: Fundibes.

RADRIGÁN, M. (coord), BUCHELI, M., PEREIRA, L. et al. (2012). La Economía social en España, Iberoamérica y países del Magreb”, en Chaves, R. Y Perez De Uralde, J.M. (dir), op. cit., Capítulo 3.

RADRIGÁN, M. (coord.) (2011). Manual de buenas prácticas de la economía social y el empleo en América Latina, Valencia : Ed. Fundación Divina Pastora y Fundibes.

SALAMON, L.M. y ANHEIER, H.K. (1997). Defining the nonprofit sector - a crossnational analysis, Institute for Policy Studies, The Johns Hopkins University.

SINGER, P. (1997). Economia solidaria: geraçao de renda e alternativa ao liberalismo, Revista proposta, $\mathrm{n}^{\mathrm{o}} 72$, p. 5-13.

TOMAS-CARPI, J.A. (1997). The prospects for a Social Economy in a changing world, Annals of Public and Cooperative Economics, Vol. 68, $\mathrm{n}^{\circ}$ 2, p. 247-279.

UNITED NATIONS (2003). Handbook on Non Profit Institutions in the System of National Accounts. Series F, No. 91. New York: United Nations (Department of Economic and Social Affairs - Statistics Division).

VIENNEY, C. (1994). L'Economie sociale, Paris : La Découverte.

VIVET, D. y THIRY, B. (2000). Field of study, quantitative importance and national acceptations, en: CIRIEC (2000), op. cit. Capítulo 1. 
WEISBROD, B.A. (1988). The Nonprofit Economy, Mass., Cambridge: Harvard University Press.

http://www.aciamericas.coop

http://www.mercosur.coop

http://www.oibescoop.coop 